\title{
Impactos psicológicos da mastectomia decorrente do câncer de mama na vida da mulher
}

\author{
Psychological impacts of mastectomy due to breast cancer in women's lives
}

Impactos psicológicos de la mastectomía por cáncer de mama en la vida de las mujeres

Nathalia Di Mase Salgado ${ }^{1 *}$, Felipe Rodrigues Figueiredo Silva ${ }^{2}$, Jamille Chalfoun Flores de Souza ${ }^{3}$, Jaqueline Maria de Azevedo Chagas ${ }^{4}$, Letícia Lourenço Botelho ${ }^{5}$, Letícia Santana Ferreira Gonçalves $^{6}$, Marília Medeiros de Matos ${ }^{7}$, Mateus Gonçalves de Sena Barbosa ${ }^{8}$, Maria Luísa Barros Quintão Couto Parreira9 .

\section{RESUMO}

Objetivo: Abordar a saúde psicológica das mulheres diagnosticadas com câncer de mama após mastectomia. Revisão bibliográfica: $O$ câncer de mama é o tumor que mais acomete a população do sexo feminino e apresenta altos índices de incidência anuais e tem em seu prognóstico precoce e consequente tratamento breve melhores desfechos, porém como a maioria dos casos é diagnosticado em fases mais avançadas em nosso país, os tratamentos são mais agressivos, como isso, a evolução da doença e suas consequências na vida das mulheres acometidas por esta patologia são características a serem abordadas neste artigo, levando em consideração as seguintes categorias de análise: (1) Estresse psicológico após mastectomia; (2) Impactos sociais e sexuais; (3) Benefícios da reconstrução mamária. Considerações finais: O processo pós mastectomia deve ser melhor elucidado para todas as mulheres que passam por esta operação. Tal abordagem deve ser realizada tanto pelo profissional que realizou o procedimento quanto pelo sistema de saúde ao qual essa paciente está vinculada, de forma a minimizar os impactos físicos e psicológicos decorrentes desta intervenção e acelerar o processo de readaptação social.

Palavras-chave: Mastectomia, Saúde da mulher, Saúde mental.

\begin{abstract}
Objective: Address the psychological health of women diagnosed with breast cancer after mastectomy. Bibliographic review: Breast cancer is the tumor that most affects the female population and has high annual incidence rates and its early prognosis and consequent brief treatment have better outcomes, but as most cases are diagnosed at more advanced stages in our country, treatments are more aggressive, therefore, the evolution of the disease and its consequences in the lives of women affected by this pathology are characteristics to be addressed in this article, taking into account the following categories of analysis: (1) Psychological stress after mastectomy; (2) Social and sexual impacts; (3) Benefits of breast reconstruction. Final considerations: The post mastectomy process should be better elucidated for all women who undergo this surgery. Such an approach must be performed both by the professional who performed the procedure and by the health system to which this patient is linked, in order to minimize the physical and psychological impacts resulting from this intervention and accelerate the process of social readaptation.
\end{abstract}

Key words: Mastectomy, Women's health, Mental health.

${ }^{1}$ Centro Universitário das Américas (FAM), São Paulo - SP. *E-mail: nat.di.mase@gmail.com

2 Universidade Federal de Minas Gerais (UFMG), Belo Horizonte - MG.

3 Universidade Iguaçu (UNIG), Itaperuna - RJ.

${ }^{4}$ Universidade de Rio Verde (UniRV), Rio Verde - GO.

${ }^{5}$ Centro Universitário de Valença (UNIFAA), Valença - RJ.

${ }^{6}$ Centro Universitário do Planalto Central Apparecido dos Santos (UNICEPLAC), Brasília - DF.

${ }^{7}$ Centro Universitário FACISA (UNIFACISA), Campina Grande - PB.

${ }^{8}$ Centro Universitário Atenas (UNIATENAS), Passos - MG.

${ }^{9}$ Centro Universitário de Caratinga (UNEC), Caratinga - MG. 


\section{RESUMEN}

Objetivo: Abordar la salud psicológica de las mujeres diagnosticadas con cáncer de mama después de una mastectomía. Revisión bibliográfica: El cáncer de mama es el tumor que más afecta a la población femenina y tiene altas tasas de incidencia anual y su pronóstico temprano y consecuente tratamiento breve tienen mejores resultados, pero como la mayoría de los casos se diagnostican en estadios más avanzados en nuestro país, los tratamientos son más agresivos. por tanto, la evolución de la enfermedad y sus consecuencias en la vida de las mujeres afectadas por esta patología son características que se abordarán en este artículo, teniendo en cuenta las siguientes categorías de análisis: (1) Estrés psicológico tras mastectomía; (2) Impactos sociales y sexuales; (3) Beneficios de la reconstrucción mamaria. Consideraciones finales: El proceso posterior a la mastectomía debería aclararse mejor para todas las mujeres que se someten a esta operación. Dicho abordaje debe ser realizado tanto por el profesional que realizó el procedimiento como por el sistema de salud al que está vinculado este paciente, con el fin de minimizar los impactos físicos y psicológicos derivados de esta intervención y acelerar el proceso de readaptación social.

Palabras clave: Mastectomía, Salud de la mujer, Salud mental.

\section{INTRODUÇÃO}

É alarmante a incidência de câncer de mama entre a população feminina contemporânea, sendo que uma em cada dezoito mulheres desenvolvem câncer de mama antes dos oitenta anos (IZYDORCZYK B, et al., 2018). Além disso, o câncer de mama é a doença maligna mais frequente entre as mulheres em todo o mundo (SALOMON MFB, et al., 2015).

Grandes avanços já ocorreram nos últimos anos em relação aos tratamentos para essa enfermidade, de modo que proporcionaram uma elevação da taxa de sobreviventes da doença. Contudo, ultimamente, a saúde psicológica tem chamado bastante atenção, visto que essa está diretamente associada à qualidade de vida e muitas mulheres sofrem grandes impactos psicológicos após a mastectomia, em virtude da necessidade para tratar o câncer de mama. Muitos estudos já demonstraram que as mulheres com câncer de mama tratadas com mastectomia possuem maiores chances de desenvolverem imagem corporal ruim, qualidade de vida mais baixa e distúrbios emocionais variados, em destaque a depressão e ansiedade (ZHANG C, et al., 2018).

Esse estresse psicológico é um componente responsável pela redução do bem-estar mental e produz um impacto extremamente negativo no rumo e na eficiência do tratamento do paciente. Tal problemática se explica por conta da associação da mastectomia a um evento traumático, provocando uma condição de estresse psicológico (GODOY MK, et al., 2016; IZYDORCZYK B, et al., 2018; GOMES NS, et al., 2015).

Em contrapartida, determinados estudos apresentaram que as mulheres pós-mastectomia e após a reconstrução mamária, prezam mais a qualidade de vida e se sentem mais encantadoras do que as mulheres mastectomizadas sem reconstrução (CAMMAROTA MC, et al., 2019; IZYDORCZYK B, et al., 2018). O objetivo deste estudo foi revisar a literatura e elucidar acerca do impacto psicológico da mastectomia decorrente do câncer de mama na vida da mulher, de modo que se ressaltam as principais evidências científicas sobre tal assunto.

\section{REVISÃO BIBLIOGRÁFICA}

O tumor que mais acomete mulheres no Brasil e no mundo é o câncer de mama, sendo responsável por quase 1 entre 4 casos novos de câncer na população feminina (FIREMAN KM, et al., 2018; ROSA D, et al., 2020). Representa, anualmente, cerca de $25 \%$ dos casos novos, sendo estimados nacionalmente 57.960 casos novos, em 2016, e 14.206 mortes femininas, em 2013. Ajustada pela população mundial, o câncer de mama apresenta uma curva ascendente, tornando-se a primeira causa de morte na população feminina brasileira (MARTINS JOA, et al., 2020).

Cerca de 2 milhões de novos casos de câncer de mama foram estimados mundialmente em 2018, já no Brasil a estimativa foi de aproximadamente 60 mil casos novos para o mesmo período. Estudos 
epidemiológicos revelaram que os índices de câncer de mama em países de baixa e média renda elevaramse velozmente nas últimas décadas. A mortalidade por câncer de mama na América Latina cresceu nos últimos 20 anos com sobrevida 20\% menor em relação à Europa Ocidental e aos Estados Unidos. Dentre as causas mais prováveis podemos citar as baixas taxas de rastreamento, estágio clínico avançado no diagnóstico e falta de acesso a tratamento adequado para a maioria dos doentes (ROSA D, et al., 2020).

A partir dos 35 anos de idade, a incidência do câncer de mama é maior, sendo raro em idades menores (MARTINS JOA, et al., 2020). Estudos demonstraram que aproximadamente $20 \%$ dos casos de câncer de mama na América Latina ocorreram em mulheres com menos de 45 anos, em divergência com taxas de 12\% em países desenvolvidos. Além do mais, a idade média relatada no diagnóstico é de 40 a 50 anos nos países asiáticos e africanos e de 60 a 70 anos nos países ocidentais (ROSA D, et al., 2020).

O prognóstico está diretamente relacionado com o diagnóstico precoce e o início do tratamento (MARTINS JOA, et al., 2020). Entretanto, no Brasil o câncer de mama é diagnosticado em fases mais avançadas, consequentemente os tratamentos são mais agressivos interferindo negativamente na qualidade de vida através de sequelas funcionais e psicológicas (FIREMAN KM, et al., 2018). Dessa forma, comparado a população em geral, a prevalência de depressão em pacientes com câncer é três vezes mais alta, estando relacionada com a piora da qualidade de vida somada ao trauma em relação à mutilação e à distorção da autoimagem devido a mastectomia (FIREMAN KM, et al., 2018).

\section{Estresse psicológico após a mastectomia}

A mastectomia é um procedimento potencialmente traumático no que tange ao impacto psicológico gerado, em decorrência de seu caráter invasivo e de sua capacidade de alterar a imagem corporal dos pacientes. Somada aos demais desafios intrínsecos à luta contra o câncer, a mastectomia colabora para o desenvolvimento de distúrbios psicológicos persistentes, como a ansiedade, o transtorno do estresse póstraumático e a depressão (IZYDORCZYK B, et al., 2018).

Para alguns autores, um dos principais pontos de estudo nesta temática é o impacto provocado pela alteração corporal dos pacientes. Após a cirurgia de retirada total ou parcial de uma ou ambas as mamas, os indivíduos enfrentam um conflito entre a forma como eles enxergavam-se antes do procedimento, forma esteticamente positiva, e a forma como encontram-se após. Essa divergência, associada aos padrões socialmente impostos, gera uma tensão capaz de agravar tendências à baixa autoestima, levando ao surgimento de sintomas depressivos e à queda da qualidade de vida (SOUZA NHA, 2017; ALKAFF TM, 2019; ZHANG C, 2018).

Os estudos indicam que o impacto psicológico da mastectomia é diretamente proporcional ao grau de radicalismo da cirurgia. Dessa forma, cirurgias mais invasivas provocam maiores impactos psicológicos, sendo a depressão o mais marcante. $O$ impacto ainda é maior em mulheres jovens com menos de 40 anos de idade. Alguns fatores podem estar relacionados a isso, tais como maiores inseguranças com relação à autoimagem, maior influência de padrões sociais de beleza e a concepção de que essa faixa etária corresponde ou, ao menos, deveria, ao ápice da beleza. Assim, quando esses ideais não são atendidos, muitas mulheres jovens sentem com maior intensidade as alterações corporais advindas da mastectomia (NASCIMENTO KTS, et al., 2015; SHERMAN KA, et al., 2016).

Alguns estudos ressaltam, no entanto, que a ocorrência de câncer de mama e a posterior mastectomia não excluem a ocorrência do chamado Crescimento Pós-traumático (CPT). O CPT é o desenvolvimento de novos elementos pessoais após um trauma, tais como força pessoal, melhores relações interpessoais, maior valorização e aproveitamento da vida e desenvolvimento espiritual. Assim, embora a mastectomia esteja fortemente associada a inúmeros distúrbios psicológicos e à deterioração da qualidade de vida, não se pode omitir a possibilidade de desfechos positivos após o procedimento (IZYDORCZYK B, et al., 2018).

\section{Impacto nos relacionamentos}

A pesquisa descritiva de abordagem qualitativa "Percepção das mulheres sobre sua funcionalidade e qualidade de vida após a mastectomia", avaliou a ação da fisioterapia aliada a percepção de vinte e nove 
pacientes mulheres, que realizaram uma mastectomia radical, com esvaziamento axilar. Essas mulheres apresentaram restrição de amplitude no movimento de membros superiores. Nesse estudo, as pacientes realizaram dez atendimentos de fisioterapia em grupo e ao final da última sessão foram encaminhadas a uma entrevista. Essas mulheres informaram que apresentaram um progresso na sua capacidade funcional, emocional e da autoestima, resultando dessa forma em uma reinserção social, assim como o retorno de atividades diárias. Pode-se assim entender que a reabilitação utilizada trouxe resultados positivos na qualidade de vida, funcionalidade, relações afetivas e sociais na vida das mulheres (IZYDORCZYK, B. et al, 2018).

Em um hospital Chinês foi realizado um estudo experimental controlado e randomizado, com uma amostra de trinta mulheres chinesas, que perderam a mama devido a um câncer de mama. Neste estudo foi analisado e comparado a relação do tipo de prótese mamária utilizada com os efeitos físicos, sociais e psicológicos das pacientes. Se fez evidenciar que essas mulheres ficaram satisfeitas com a prótese mamária de temperatura controlada, mas preferem as autoadesivas, apesar da sua limpeza ser um empecilho. Independente da prótese utilizada, ela restaura a credibilidade social, o senso de pertencimento, melhora o desempenho no esporte, eleva a autoestima e confiança da mulher. Entretanto, há limitações com o resultado desse estudo, pois a amostra utilizada é restrita, assim como a localização geográfica, que associada a realidade de um único centro não pode ser generalizada e aplicada em toda a China (QIU J, et al., 2020).

\section{Impacto na autoestima}

O impacto na autoestima de mulheres mastectomizadas pode requerer intenso tratamento psicológico para o restabelecimento e aceitação de sua nova imagem corporal. Devido a diversas consequências de seu tratamento, o desenvolvimento de depressão no pós-operatório é uma das ocorrências mais comuns. Foi demonstrado que, entre pacientes portadoras de câncer de mama submetidas à mastectomia radical, há elevada prevalência de quadros depressivos de variadas intensidades. Dessa forma, após uma cirurgia de retirada das mamas, a mulher pode apresentar uma série de desafios no processo de retomar sua autonomia e sua rotina profissional, social, familiar e sexual. Logo, o acompanhamento de um psicólogo, juntamente com o de um psiquiatra, faz-se de grande importância em associação com o apoio e acolhimento das pessoas do convívio diário, como familiares e amigos (GODOY MK, et al., 2016).

\section{Impacto na sexualidade}

A mastectomia pode proporcionar deformações na imagem corporal, identidade e autoestima da paciente, podendo repercutir em sua sexualidade. As mamas são uma parte do corpo feminino que tem muita relevância no aspecto sexual. Assim, sua retirada pode gerar danos no desempenho sexual. Vale ressaltar que $o$ ato sexual transcende a reprodução, tendo o sexo também como objetivo o prazer. Qualquer alteração em suas fases pode ser considerada disfunção sexual (MARTINS JOA, et al., 2020).

Desde o diagnóstico da doença, a mulher com câncer de mama já tem sua qualidade sexual afetada, visto que a sua autoestima fica abalada, começa a ter medo da morte e os tratamentos mais convencionais, como quimioterapia e radioterapia, também geram modificações no corpo feminino: atrofia vulvovaginal, dispareunia, irritação e secura vaginal, inibição do desejo ou excitação, menopausa prematura, náuseas e vômitos (SILVA LM, 2016). Ademais, algumas mulheres se sentem mutiladas em decorrência da retirada da mama e apresentam dificuldades em expor seu corpo aos seus parceiros, visto que para mulher a imagem corporal é algo com grande significado. Assim, a perda desse órgão gera uma repercussão física e psíquica, trazendo prejuízo para sua qualidade de vida, na satisfação sexual e recreativa (OLIVEIRA FBM, et al., 2017).

Uma das formas de avaliar a atividade sexual, é por meio do questionário Quociente Sexual - Versão Feminina (QS-F), desenvolvido no Programa de Estudos em Sexualidade (ProSex) do Instituto de Psiquiatria do Hospital das Clínicas da Faculdade de Medicina da Universidade de São Paulo; porém, é um questionário antigo e pouco conhecido entre os profissionais da saúde. Estudos usando o QS-F revelaram que as mulheres jovens enfrentam maiores dificuldades na vida sexual após a mastectomia, e que $50 \%$ das entrevistadas identificaram-se com alguma disfunção sexual, sendo o desejo sexual hipoativo a mais frequente (MARTINS JOA, et al., 2020). 
Um estudo realizado com 20 mulheres, em Cingapura, mostrou que 9 apresentaram problemas no relacionamento conjugal após serem submetidas à cirurgia, como a diminuição na frequência sexual. Além da mudança no desempenho sexual, algumas mulheres evitavam se despir para o parceiro, não permitiam serem tocadas ou utilizavam camisetas durante as relações (SILVA LM, 2016). Analisando os diversos estudos, foi possível observar que a maioria das pesquisas sobre o câncer de mama não é focada em investigar o impacto deste na vida sexual, visto que os profissionais de saúde não costumam discutir como está a sexualidade da paciente após o tratamento para o câncer (MARTINS JOA, et al., 2020).

Portanto, é importante que os profissionais de saúde que assistem às pacientes e que vivenciam o contexto do adoecimento por câncer de mama, se atentem para as consequências geradas pelo procedimento cirúrgico (SILVA JAG, 2014). Uma forma prática de se identificar os impactos da cirurgia é aplicando um questionário com algumas perguntas referentes ao tema. Desta forma, o profissional de saúde terá uma melhor percepção das mudanças ocorridas no pós-cirúrgico, podendo ajudar a paciente a adaptar sua rotina com o menor impacto possível na sua qualidade de vida (OLIVEIRA FBM, et al., 2017; MARTINS JOA, et al., 2020).

\section{Os benefícios da reconstrução mamária}

A possibilidade de recuperação da mama tem a capacidade de devolver a autoestima da mulher que enfrentou o câncer. Nota-se que, além da função de amamentação, a mama é considerada relevante para construção da autoimagem, sendo que a reconstrução ajuda a muitas mulheres a aceitar novamente a imagem do corpo e, consequentemente, superar a retirada em decorrência da cirurgia. Por certo, para muitas mulheres a reconstrução mamária está intimamente relacionada à restauração da autoestima e feminilidade, além de proporcionar uma nova dedicação aos cuidados estéticos (INOCENTI A., et al, 2016).

Em virtude de um maior número de casos de câncer de mama, a busca pela reconstrução mamária também vem crescendo e, em consonância, a preocupação em relação a satisfação proveniente das pacientes. Dessa maneira, as técnicas de reconstrução são variadas mediante a cirurgia realizada para cada tratamento em específico (SHERMAN KA, et al., 2016).

No Brasil, sabe-se que as técnicas de reconstrução mamárias englobam procedimentos como: reconstrução com Retalho do Músculo Reto Abdominal (RMRA) e com retalho do músculo grande dorsal, sendo utilizado um expansor tecidual que subsequentemente será substituído por uma prótese de silicone (COSTA AMN, et al., 2015). O processo de reconstrução mamária tem por finalidade recuperar a estética corporal e, por consequência, melhorar a autoimagem da paciente, promovendo a restauração do volume que foi anteriormente perdido e garantindo a simetria com a mama oposta (CAMMAROTA MC, et al., 2019).

Estudos realizados com pacientes do sexo feminino submetidas à reconstrução mamária, demonstraram que parte das pacientes apresentou melhora significativa na qualidade de vida, além de estarem satisfeitas no aspecto psicológico e relações sociais. Alguns pacientes são submetidos à mastectomia bilateral e com isso, verifica-se a existência de uma eficácia positiva da reconstrução mamária bilateral, em decorrência da simetria (MARTINS TNO, et al., 2017).

Existem alguns fatores que estão relacionados com a reconstrução mamária como à idade, raça, nível socioeconômico, estágio e prática médica. Idade inferior a 50 anos foi um preditor constante e melhor de reconstrução mamária (NOAL SJ e BERGAMASCHI DA, 2017). Oliveira MCM, et al. (2013), afirmam que existe um receio na realização de procedimentos cirúrgicos em pacientes mais idosas, apesar disso, a reconstrução da mama é uma opção segura e viável em pacientes mais velhas. Dessa maneira, a idade biológica da paciente e as comorbidades precisam ser consideradas. Contudo, mulheres de todas as idades com câncer de mama devem receber reconstrução mamária (COSAC OM, 2013).

A realização da reconstrução da mama após a mastectomia aumentou lentamente na última década e deve-se atentar que a cirurgia do câncer de mama trata-se de um procedimento complexo em que a tomada de decisão irá envolver a paciente, o cirurgião e o cirurgião plástico (GOMES NS, et al, 2015). Por isso, o paciente tem o direito de escolher a alternativa que melhor desejar e deverá, portanto, estar ciente acerca de todas as opções disponíveis, bem como complicações e benefícios (INOCENTI A, et al, 2016). Diante de um 
procedimento complexo, é necessário salientar que a mastectomia causa desestabilização psicológica na paciente e que, em contrapartida, a reconstrução mamária é capaz de trazer benefícios que incluem, principalmente, a melhora da imagem corporal, autoestima, bem-estar e uma possível vantagem de sobrevivência (HOWARD-MCNATT MM, 2013).

\section{CONSIDERAÇÕES FINAIS}

De acordo com as informações apresentadas nessa revisão, evidenciou-se que o processo de mastectomia causa à mulher (i) alterações físicas e (ii) psicológicas, em que se torna fundamental que o profissional de saúde compreenda a mulher como um todo, fornecendo a ela informações acerca dos impactos causados pelo procedimento realizado. Os artigos revisados demonstram a importância da cirurgia de reconstrução, sendo um direito que deve ser exigido junto ao SUS e aos planos de saúde para que essas mulheres tenham melhor qualidade de vida após danos físicos e psicológicos causados pela mastectomia. Sendo assim, esse estudo compila de forma direcionada o ponto de vista imposto pela mastectomia e pela reconstrução mamária, contribuindo para um entendimento holístico acerca da temática.

\section{REFERÊNCIAS}

1. ALKAFF TM, et al. Awareness, acceptance, and perspective of women for reconstruction post mastectomy. The Journal of the Pakistan Medical Association, 2019; 69(1) : 141-155.

2. CAMMAROTA MC, et al. Quality of Life and Aesthetic Results after Mastectomy and Mammary Reconstruction. Revista Brasileira de Cirurgia Plástica (RBCP) - Brazilian Journal of Plastic Surgery, 2019; 32(1): 45-57.

3. COSAC OM, et al. Reconstrução Mamária de Resgate: A Importância Dos Retalhos Miocutâneos. Revista Brasileira de Cirurgia Plástica, 2013; 28: 1.

4. COSTA AMN, et al. Mulheres e a mastectomia: revisão literária. Revista de Atenção à Saúde, 2015; $13:$ 23-37.

5. FIREMAN KM, et al. Percepção das Mulheres sobre sua Funcionalidade e Qualidade de Vida após Mastectomia, Revista Brasileira de Cancerologia 2018; 64(4): 499-508.

6. GODOY MK, et al. Mastectomia e estética corporal: uma revisão. Salão do Conhecimento, 2016; 2(3): 15-22.

7. GOMES NS, et al. Autoestima e qualidade de vida de mulheres submetidas a cirurgia oncológica de mama. Revista Mineira de Enfermagem, 2015; 19(2) : 33-45.

8. HOWARD-MCNATT MM. Patients Opting for Breast Reconstruction Following Mastectomy: An Analysis of Uptake Rates and Benefit. Breast Cancer: Targets and Therapy, 2013; 5(1) : 9-15.

9. INOCENTI A, et al. Repercussão dos efeitos da cirurgia reconstrutora na vida de mulheres com neoplasias de mama. Revista Texto Contexto Enfermagem, 2016; 25 (2): 1-9.

10. IZYDORCZYK B, et al. Psychological Resilience as a Protective Factor for the Body Image in Post-Mastectomy Women with Breast Cancer. International Journal of Environmental Research and Public Health, 2018; 15(6): 1181.

11. MARTINS JOA, et al. Sexualidade de Mulheres Submetidas À Mastectomia: Identificação das Fases Afetadas no Ciclo da Resposta Sexual, R. pesq.: cuid. fundam. online 2020 jan/dez 12: 67-72.

12. MARTINS TNO, et al. Reconstrução mamária imediata versus não reconstrução pós-mastectomia: estudo sobre qualidade de vida, dor e funcionalidade. Fisioterapia Pesquisa, 2017; 24(4): 412-419.

13. NASCIMENTO KTS, et al. Sentimentos e fontes de apoio emocional de mulheres em pré-operatório de mastectomia em um hospital-escola. Revista de Enfermagem da UERJ, 2017; 23(1) : 12-15.

14. NOAL SJ, BERGAMASCHI DA. Sentimentos experimentados por mulheres que realizaram mastectomia. Anuário Pesquisa e Extensão Unoesc São Miguel do Oeste, 2017;2: 20-30.

15. OLIVEIRA FBM, et al. Impacto do câncer de mama e da mastectomia na sexualidade feminina. Revista de Enfermagem da UFPE, 2017.

16. OLIVEIRA MCM, et al. Autoestima, depressão e espiritualidade em pacientes submetidas a mastectomia ou quadrantectomia com linfadenectomia axilar. Revista do Médico Residente, 2013; 15(3): 21-23.

17. QIU J, et al. Physical and psychological effects of different temperature-controlled breast prostheses on patients with breast cancer during rehabilitation:a randomized controlled study (CONSORT). Medicina, 2020; 99(13).

18. ROSA DD, et al. The impact of sociodemographic factors and health insurance coverage in the diagnosis and clinicopathological characteristics of breast cancer in Brazil: AMAZONA III study (GBECAM 0115), Breast Cancer Res Treat, 2020;183(3):749-757

19. SALOMON MFB, et al. Câncer de mama no homem. Rev Bras Mastologia. 2015; 25(4):141-5

20. SHERMAN KA, et al. Body image and psychological distress in nipple-sparing mastectomy: the roles of selfcompassion and appearance investment. Wiley Online Library, 2016; 17( 3): 21-23.

21. SILVA JAG. Cartilha câncer é preciso falar disso, fatores de risco ambientais e hormonais. INCA. Instituto Nacional de Câncer, 2014; 4(3): 14-23.

22. SOUZA NHA, et al. Câncer de mama em mulheres jovens: um estudo epidemiológico no nordeste brasileiro. Revista Sanare, 2017;16(2).

23. ZHANG C, et al. Depression Induced by Total Mastectomy, Breast Conserving Surgery and Breast Reconstruction: $A$ Systematic Review and Meta-analysis. World J Surg, 2018; 42(7): 2076-2085. 